\title{
Caffeine dependence: An alternative view
}

To the editor - A recent article ${ }^{1}$ has discussed the issue of caffeine dependence. Several different claims can be made regarding caffeine dependence, and it is essential to carefully evaluate the evidence relating to these. One view would suggest that it is a global phenomenon affecting a large percentage of the population. I suggest that this is not the case, as many studies show that symptoms and signs of withdrawal are selective and restricted to only a sample of subjects $^{2-5}$. Another view, for which there is stronger evidence, is that certain individuals report caffeine dependence and that withdrawal is associated with headache and mood changes. These sensitive individuals are also able to discriminate very low levels of caffeine. It is not surprising, therefore, that when they are put in the context of a caffeine withdrawal study, they report more symptoms when they can ascertain that they are drinking decaffeinated coffee.

Support for the above view comes from results from a study on withdrawal of caffeine. One hundred and forty-four students took part in a double-blind experiment examining the effects of caffeine withdrawal. Half of the subjects were given caffeinated instant coffee to drink for six days and the others decaffeinated coffee (and decaffeinated tea). During this period the subjects were required to consume coffee made only from these supplies and to abstain from any other caffeinated products. Records were kept to assess compliance. To chart any changes in physical health and mood, a symptom checklist and mood rating was filled out by all subjects at 6 p.m. each day during the test week. The subjects in the caffeinated and decaffeinated groups were well-matched in terms of age, gender, weight, alcohol consumption, educational level, personality and regular level of caffeine consumption (median $=195 \mathrm{mg}$, calculated from a one-week diary). It is also important to note that the subjects were not good at discriminating between caffeinated and decaffeinated coffee (they were almost at chance level). Interest here focuses on the headache data, which are shown in the figure. If one first considers those given decaffeinated coffee, the well-established finding of an increase in the reporting of headaches over the first three days of caffeine withdrawal is seen. However, the group given caffeinated coffee also showed an increase in the number of reported headaches. These results can be interpreted as an expectancy effect to report headaches when you think you are having caffeine withdrawn. In previous studies it is possible that the subjects could detect the caffeine in the caffeinated coffee and that this removed the expectancy effect. In the present study the subjects could not reliably detect the caffeine, which plausibly explains why both groups show an increase in headaches.

Although the above explanation is clearly very different from one based on physiological dependence, I am not suggesting that one view is completely right and the other wrong. Rather, these are alternative views that must be investigated further. Again, this is very different from the view advocated by Pickworth'. Indeed, there are other points in his article that must be questioned and need to be examined in further studies. For example, he argues that labeling caffeine as dependenceproducing may result in a reduction in its use but that this will have no consequences. He states that it is rarely used medically, which is clearly incorrect if one includes over-the-counter medication. There is also another area where reduction of caffeine use may lead to negative effects. This is mental efficiency where it has been clearly demonstrated that caffeine improves the ability to sustain attention, especially when arousal is $l o w^{6,7}$. Failure to use this means of maintaining performance may, in the majority of caffeine users, have far greater consequences than any risks of dependence.

Overall, there is little evidence for caffeine dependence being a general effect seen at all levels of caffeine consumption. There is a stronger case for the view that certain individuals are caffeine dependent, although even here it is unclear whether the caffeine is causal or whether sensitivity to caffeine and its withdrawal merely reflects the presence of existing problems. Large-scale epidemiological investigations and research aimed at

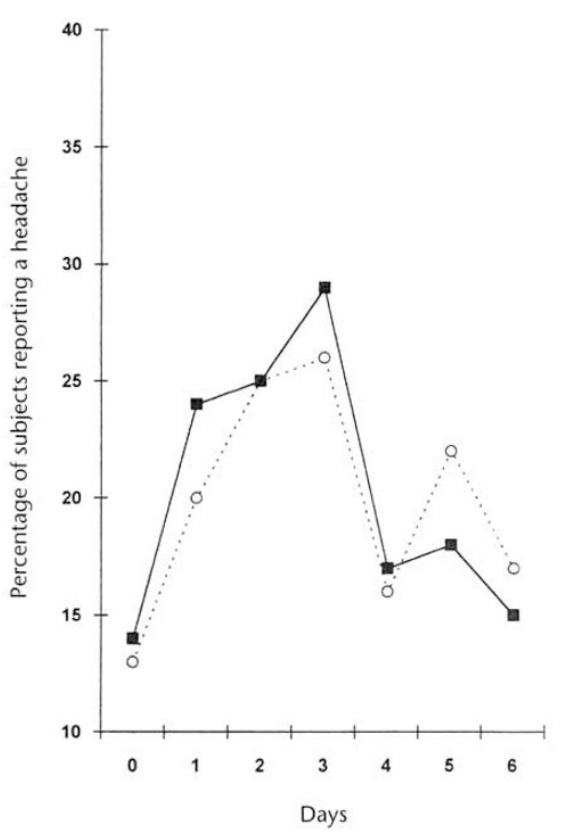

Percentage of subjects receiving caffeinated and decaffeinated coffee reporting a headache over the 7 days of the study. (Day 0 , day before control of usage; day 6 , last day drinking designated coffee.) 0 , Caffeinated coffee; $\boldsymbol{\square}$, decaffeinated coffee.

elucidating the mechanisms of caffeine actions in the brain will lead to a better understanding of these issues.

The author's research has received support from the coffee industry.

\section{ANDREW P. SMITH}

Department of Psychology

University of Bristol

8 Woodland Road

Bristol BS8 1TN, UK

1. Pickworth, W.B. Caffeine dependence. Lancet 345, 106 (1995).

2. Silverman, K., Evans, S.M., Strain, E.C. \& Griffiths, R.R. Withdrawal syndrome after double-blind cessation of caffeine consumption. N. Engl. J. Med. 327, 1109-1114 (1992).

3. van Dusseldorp, M. \& Katan, M.B. Headache caused by caffeine withdrawal among moderate coffee drinkers switched to ordinary decaffeinated coffee: A 12-week double blind trial. $\mathrm{Br}$. Med. J. 300, 1558-1559 (1990).

4. Griffiths, R.R. et al. Low-dose caffeine physical dependence in humans. J. Pharmacol. Exp. Ther. 225, 1123-1132 (1990).

5. Strain, E.C., Mumford, G.K., Silverman, K. \& Griffiths, R.R. Caffeine dependence syndrome. JAMA 272, 1043-1048 (1994).

6. Smith, A.P., Brockman, P., Flynn, R., Maben, A. \& Thomas, M. Investigation of the effects of coffee on alertness and performance during the day and night. Neuropsychobiology 27, 217-223 (1993).

7. Smith, A.P. Caffeine, performance, mood and states of reduced arousal. Pharmacopsychoecologia 7, 75-86 (1994). 